\title{
Definitions, Summary, and Some Issues
}

\author{
Peter S. Conti \\ JILA and APS Department, University of Colorado, Boulder CO 80309
}

\section{Definitions}

This Joint Discussion has been titled Massive Star Birth. Perhaps it is appropriate here to define what we mean by a massive star. The very word massive suggests we consider a minimum mass $M$ below which one would speak of low (or intermediate) mass evolution, and above which is the realm of massive stars. It is natural to take this mass limit as that in which a (single) star will end its life as a supernova: $8 M_{\odot}$. This corresponds to a (minimum) luminosity $L$ of a few $\times 10^{3} L_{\odot}$, a (minimum) $T_{\text {eff }}$ of $20000 \mathrm{~K}$, and a ZAMS spectral type of about B1.5V. Note that this mass division refers to the final evolution of a star, and might well have nothing to do with difference in physical processes between massive and low mass star birth. For example, the minimum $T_{\text {eff }}$ for a star to produce an UCHII region, a readily observable quantity, corresponds to a $T_{\text {eff }}$ closer to $30000 \mathrm{~K}$ and a mass of $15 M_{\odot}$. On the other hand, the UCHII phase is time dependent during the stellar birth processes and its absence would not necessarily be a good minimum mass indicator. Maeder has noted that an important distinction between high and low mass star formation is whether or not the accretion time scale is larger or smaller than the "Kelvin-Helmholz" time, respectively. He suggests that this point is near $8 M_{\odot}$. Finally, in a whimsical spirit I suggest that massive stars throughout their birth processes be referred to as Massive YSOs, or MYSOs, to be pronounced as "my sos".

\section{Summary}

Massive star birth, unlike nearly all other fields of stellar astrophysics, is nearly entirely hidden from view in the visible but amenable to observation at IR, $\mathrm{mm}$, and $\mathrm{cm}$ wavelengths. This JD was roughly organized so that the earliest phases of massive star birth were discussed first, followed by later stages, ending with the emergence of a hot star from its dust cocoon and optical visibility. Williams stated that massive stars form in clusters within self-gravitating molecular clouds. He considered the conditions needed for the formation of massive stars and concluded that turbulence probably plays a leading role. As this quantity dissipates the cloud(s) can begin to collapse. Mundy's talk was concerned with the environment of embedded massive stars. This material radiates strongly in $\mathrm{mm}$ and sum-mm regions. He pointed out that the initial masses involved in massive star formation are much larger than what eventually ends up in the star. This very substantial non-accreted material is somehow ejected in bipolar outflows, eventually forming low mass stars which are typically associated with massive stars in a cluster. Van Dishoeck \& van der Tak discussed spectroscopy 
of the complex molecules that are found during initial formation stages. There are a wide range of physical conditions throughout the pre-stellar envelope and various molecules sample the different spatial regions, ranging from hot cores to regions containing evaporated ices. Dyson et al. summarize the hydrodynamics of the ionization and recombination fronts. The issue of the role of magnetic fields was again raised, but answers concerning its significance are not yet in hand. Observations of the jets and outflows are detailed by Menten. Unlike low mass stars, this ejected material does not seem to be highly collimated in jets, but rather the outflows have a relatively wide opening angle. The evidence for the presence of discs in massive stars is considered and shown to be likely by Cesaroni. Here the tracers involve high velocity resolution studies of various molecular species, looking for Keplerian motion.

Churchwell pointed out the potential presence of precursor UCHII candidates from sub $\mathrm{mm}$ observations of luminous sources. He also suggested that hard X-ray point sources in star forming regions might be another indication of the initial formation stages. Lizano \& Garay gave a summary of their exquisite and detailed published review of massive star formation. Cox summarized ISO spectroscopic studies of the abundances that may be determined from the compact HII regions surrounding massive stars. Hanson gave a progress report on the theory which will be used to connect the observed properties of near IR spectral lines with stellar parameters. This can be used for stars which have emerged from their dust cocoons. More detailed observations are to be expected soon as new telescopes and better detectors come on line. Maeder showed that massive stars can grow by accretion of dust and gas in the time scales available if that rate goes as a power law, say $M^{\mathbf{1 . 5}}$ or so. He points out that this exponent has been observed in the bipolar outflow mass loss surrounding massive stars. Under the assumption that the accretion and outflow rates are correlated, this means that massive stars can grow by accretion within the current framework.

Conti \& Blum called attention to the presence of a (radio detected) cluster of O star UCHII objects in W49, a GHII region in our Galaxy. These objects are not visible in the $\mathrm{K}$ band, whereas in other GHII regions they have studied an $\mathrm{O}$ star cluster is clearly seen at this wavelength. In W49, each cluster $\mathrm{O}$ star is still contained within its birth cocoon and thus in the earliest birth stages. W49 is a less luminous, but nearby, example of buried super star clusters discovered by Johnson and by Turner. These two authors described their independent and innovative discoveries of luminous thermally excited radio regions in starburst galaxies. These objects emit optically thick free-free radio emission but are mostly invisible at optical and near IR wavelengths. These ultra-dense HII regions, or buried super star clusters, should have very strong dust emission signatures in the $\mathrm{mm}$ and sub-mm regions. These would be the youngest phases of massive cluster formation, with properties similar to but scaled up from individually excited UCHII.

\section{Some Issues}

A number of interesting questions occurred to me while sitting in on the JD concerned with massive star birth. Given that I am assigned to write this summary, I will take the opportunity to put them down (in no particular order). 
1) What happens to the mass that is not accreted by the final stellar object? As we have seen, this is $90 \%$ or more of that involved in the initial infall processes for a massive star. Exactly how does it turn into lower mass stars?

2) Are low mass stars already present as individual entities in the earliest stages of formation of massive stars? Or do they only form from the ejecta of massive objects?

3) Some O stars (and W-R types) seem to be found in our Galaxy, and elsewhere, in isolation from any other stars, or clusters, and in between spiral arms. Do these have a different formation origin? For example, not in GMCs?

4) How does one account for the formation of close binaries in the current (or any) framework? Or, for that matter, wide binaries?

5) What are the time scales for dust and ice evaporation by the massive hot star as compared to the dynamical times of the ionization front expansion and the wind dissipation of the environment? All these processes are at work in massive star formation.

It is nice to end the Proceedings of JD3 with notice of a brand new field of study, namely the advent of highly luminous counterparts to the UCHII regions, the buried super star clusters. It appears that there is a luminosity sequence in going from (more or less) individually excited UCHII regions, through W49 with some few dozen hot stars, to the buried super star clusters in starbrust galaxies with hundreds to thousands of $O$ type objects. We may be beginning to be able to study the earliest stages of cluster formation. This work, just in its infancy, is going to give an important connection to IR luminous galaxies and to cosmology, with respect to the formation of the first generations of stars.

Finally, it is clear from the enthusiasm expressed at this meeting that a larger conference is called for. Ed and I have agreed to organize a Symposium on the topic of this JD which we will propose to the IAU to be held in 2003 or 2004. In the meantime, we will also host a Workshop (Hot Stars III) in Boulder in summer of 2001 concerning massive star birth from the prospective of stellar astrophysics.

\section{References}

Names where included refer to papers presented at this Joint Discussion 